\title{
STUDY ON MICROBIAL AND PHYSICAL CHANGES IN FISH SAUCE DURING FERMENTATION
}

\author{
Md. Faisal ${ }^{1^{*}}$, Salma Noor-E-Islami ${ }^{2}$, Md. Nazrul Islam ${ }^{3}$, Md. Kamal $^{3}$ and Mohammed \\ Nurul Absar Khan ${ }^{1}$
}

${ }^{1}$ Department of Fishing and Post-Harvest Technology, Faculty of Fisheries, Chittagong Veterinary and Animal Sciences University, Chittagong-4225, Bangladesh; ${ }^{2}$ Department of Fisheries, Government of the People's of Republic Bangladesh; ${ }^{3}$ Department of Fisheries Technology, Faculty of Fisheries, Bangladesh Agricultural University, Mymensingh-2202, Bangladesh

*Corresponding author: Md. Faisal, E-mail: faisalmohammad10@gmail.com

\section{ARTICLE INFO ABSTRACT}

\section{Received}

12.05.2015

Accepted

07.06 .2015

Online

04.09.2015

Key words

Fish sauce

Fermentation

Salt treatment

Microbial changes

Physical changes
The production of fermented fish sauce was replicated in the laboratory of the Dept. of Fisheries Technology in order to study the microbiological and physical changes associated with the process. Fish sauce was produced by incubating mixtures of low valued small freshwater fish at different concentrations of sodium chloride $(25 \%$ as Treatment-I, 30\% as Treatment-II and 35\% as Treatment-III) for 9 months of fermentation (March to December) and 15 days of aging. Changes in bacterial composition and physical condition were observed. Due to effect of salt the bacterial population gradually changed and finally salt tolerant bacteria survived and multiplied. The major bacterial groups identified in fish sauce were Bacillus, Micrococcus, Lactobacillus and Pseudomonas. Due to action of enzymes present in fish body and intestine the solid fish gradually converts in to liquid where salt acts a preservative to prevent the fish from spoilage. Finally the sensory analysis of fish sauce was done through panel test. Considering the values of different parameters (salt content, test, colour and flavour) fish sauce produced with $25 \%$ salt (TreatmentI) was found to be of best quality

To cite this article: M Faisal, SNE Islami, MN Islam, M Kamal and MNA Khan, 2015. Study on microbial and physical changes in fish sauce during fermentation. Res. Agric. Livest. Fish. 2 (2): 375-383.

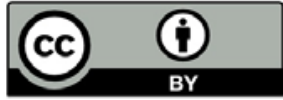

This is an open access article licensed under the terms of the Creative Commons Attribution 4.0 International License

www.agroaid-bd.org/ralf, E-mail: editor.ralf@gmail.com 


\section{INTRODUCTION}

Fermentation is one of the oldest techniques in food preservation as it not only extends the shelf-life but also enhances the flavor and nutritional quality of the product (Visessanguan et al., 2004). Fish sauce, a fermented product, is a brown, liquid seasoning commonly used in Asian countries. It has various names according to producing countries: patis in the Philippines, shotturu in Japan, budu in Malaysia, nam-pla in Thailand, nuoc-mam in Vietnam, ketjapikan or bakasang in Indonesia, yu lu in China and ngapi in Myanmar (Sanceda et al., 2003). It has been used in various prepared foods and sauce with the merit of its characteristic, favorable taste and nutritive value. It is basically produced from a mixture of fish and salt that has been allowed to ferment. During fermentation its degradation products, amino acids and peptides, have a considerable effect on the sensory characteristics of fish sauce. Enzymes from fish, bacteria or exogenous enzyme play a major role in fermentation process (Orejana, 1978; Beddows et al., 1979: Mclver et al., 1982). In Southeast Asia, fish sauce is not only popular as a condiment, but in some areas and certain social classes in the region, it is the main source of protein in the diet and has become a necessity in the household. This study, therefore, is aimed at investigating the microbiological and physical changes of fish sauce during its long period of fermentation.

\section{MATERIALS AND METHODS}

The present study was conducted in the laboratory of the Dept. of Fisheries Technology, Bangladesh Agricultural University, Mymensingh. The samples were collected from Kamal- Ranjit (KR) market of Bangladesh Agricultural University, Mymensingh in fresh condition. Fish species included were Kholisa (Colisa fasciatus), Tit punti (Puntius ticto), Jat punti (Puntius sophore), Tengra (Mystus vittatus), Taki (Channa punctatus), Gutum (Lepidocephalichthys guntea), Guchi (Macrognathus pancalus), Veda (Nandus nandus), and Bujuri (Mystus tengara). All the fish samples were of small and non-commercial size.

\section{Preparation of fish sauce}

The collected fish samples were washed thoroughly with fresh water to remove all dirt, slime, and unnecessary particles. Then the samples were weighed and divided into three parts ( $\mathrm{Kg}$ each), then mixed properly with three different concentration $(25 \%, 30 \%$ and $35 \%)$ of salt. A layer of salt was placed at the bottom of each plastic container, and then salt mixed fish were poured in it. Finally, a layer of salt was spread to cover the surface of the fishes. Plastic container with salted fish samples were covered with lids and kept in a dry place. After 7 days unsaturated brine solution was added to submerge the fish to prevent it from oxidation.

Floating fishes on the surface of brine water were kept in submerged condition by placing a weight and allowed to ferment for 9 months. The physical and microbial changes of the fish samples during fermentation were determined at 15 days intervals during this period. After 9 months the liquid part was extracted from the fermented fish samples, aged in the direct sunlight for 15 days, and filtered to separate the undigested portions. Thus the fish sauce became ready to use (Figure 1).

\section{Analytical methods}

Microbiological analysis of the fish sauce was carried out weekly during the first month and at 15 days intervals thereafter. All the representative fish species were mixed together and ground in a sterile mechanical grinder to make a paste. This ground sample was used for bacteriological analysis. For culturing bacteria, $10 \mathrm{~g}$ of fish sample was transferred aseptically to $90 \mathrm{ml}$ of sterile $0.2 \%(\mathrm{w} / \mathrm{v})$ peptone water. Serial decimal dilutions in $0.2 \%$ peptone water were prepared and $0.1 \mathrm{ml}$ of appropriate dilutions was poured on selective agar plates and spread with sterile $L$ shaped glass rods followed by incubation at $37^{\circ} \mathrm{C}$ for $48 \mathrm{hrs}$ (FAO, 1979). The isolates from the fish sauce at different stages were examined for gram stain, cell shape, motility, oxidase, catalase etc. Isolation and identification of bacteria was done according to the method suggested in Cown and Steel's Manual for the Identification of Medical Bacteria (Barrow and Felthham, 1993). Physical changes during fermentation were continuously observed based on outer appearance, texture of fish and colour of exudates. Finally the sensory evaluation was done by panel test based on colour, aroma, test and texture. Taste panel were composed of 8 internal members ( 4 men and 4 women, having age 20 to 35) who had been trained to the standard level of proficiency on panelist on sensory evaluation. 


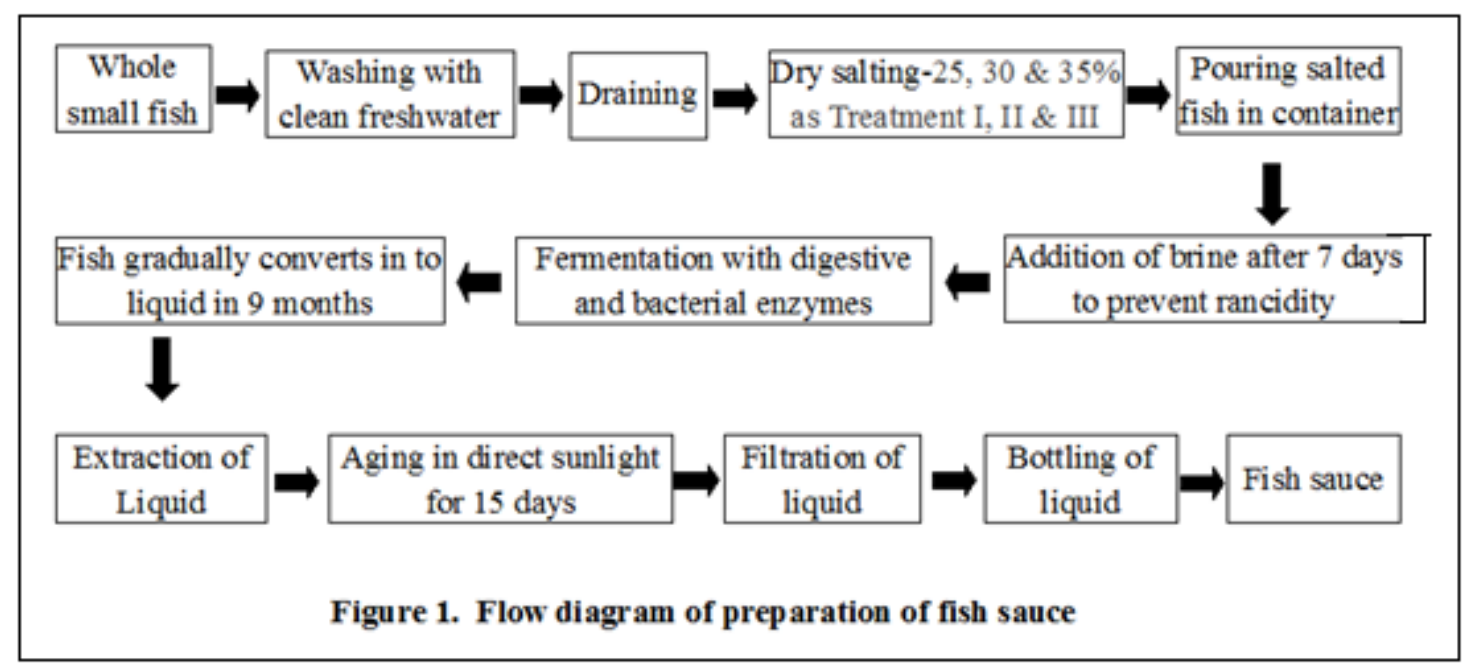

\section{RESULTS AND DISCUSSION}

\section{Generic distribution of bacteria in different treatments during fermentation}

The generic distribution of bacteria in different treatments during fermentation has been represented in Table 1. Bacillus was dominant during the whole period of fermentation, because of its spore forming and salt tolerant ability. At the initial stage Micrococcus, Lactobacillus, Corynebacterium, Escherichia .coli, Streptococcus and Pseudomonas were also present. As the fermentation progressed due to higher concentration of salt Corynebacterium, E.coli and Streptococcus failed to survive and removed. At the end of 9 months fermentation and 15 days aging in sunlight Bacillus, Micrococcus, Lactobacillus and Pseudomonas were the dominant species.

The predominance of Bacillus species in the microflora of fermented fish sauce has been reported by Saisithi et al. (1966), Crisan and Sands (1975) and Beddows (1985). However, Thongthai and Siriwongpairat (1990) demonstrated the presence of extremely halophilic bacteria identified as Halobacteria in Thai fish sauce. Sanchez (2001) stated that the microorganisms responsible for the fermentation and preservation are Lactobacillus, Streptococcus, Leuconostoc, Pediococcus, Bacillus, Debaryomyces, Pseudomonas, Alcaligenes, Rhizopus, Acetobacter, Saccharomyces, Candida, and Torulopsis spp. According to Lopetcharat and Park (2002) Staphylococcus, Bacillus and Micrococcus were found as predominant microorganisms during fermentation of fish sauce. Zaman et al. (2009) reported the presence of several microorganisms such as Enterobacteriaceae, Micrococci and Lactobacilli in fish sauce. The present study of fish sauce also agrees with the results of the above reports. According to the result of the present study there was only a little difference among the generic distribution of bacteria in three treatments.

\section{Physical changes in fish sauce during fermentation}

The physical changes in 3 (three) treatments during fermentation has been represented in Table 2 . Before addition of salt fish sample had shiny appearance and elastic texture of fresh fish. After the addition of salt within few days fishes loss shininess and gradually lost the scales, skin, flesh exposed, belly disappeared and finally skeleton become exposed. Elastic texture of fish become soft and gradually muscles become digested due to action of enzymes. Similarity, at the beginning colours of the exudates (brine) was light yellowish and gradually it became light red, dark red, dark red and turbid and finally highly turbid. Infact those physical changes are common phenomenon for the fish fermentation process. Enzymes from fish, bacteria or exogenous enzyme play a major role in fermentation process (Orejana 1978, Mclver et. al., 1982). After 9 months of fermentation fishes in 3 (three) different treatments are fully digested and liquefied. The liquid from three different treatments was collected and poured in the conical flasks separately. The mouth of each conical flask was properly sealed with aluminum foil to prevent cross contamination. 
The conical flasks were kept in the direct sunlight for 15 days for aging the liquid. When aging was completed a deep brown colour liquid aggregated at the bottom of the flasks. This deep brown colour liquid was filtered and separated by using fine meshed filters and siphoning process from the undigested parts. The filtered liquid was transferred separately in to other clean conical flasks and marked properly according to their treatments. According to Saisithi (1967) fish sauce colour varies from straw colour, amber or reddish brown. The similar result was found in this study also.

Table 1. Generic Distribution of Bacteria in 3 (three) treatments during fermentation

\begin{tabular}{|c|c|c|c|}
\hline Days & Treatment-|* & Treatment-II* & Treatment-III* \\
\hline 0 & $\begin{array}{l}\text { Bacillus, Micrococcus, } \\
\text { Lactobacillus, } \\
\text { Corynebacterium, E. coli, } \\
\text { Streptococcus and } \\
\text { Pseudomonas }\end{array}$ & $\begin{array}{l}\text { Bacillus, Micrococcus, } \\
\text { Lactobacillus, Corynebacterium, } \\
\text { E. coli, Streptococcus and } \\
\text { Pseudomonas }\end{array}$ & $\begin{array}{l}\text { Bacillus, Micrococcus, } \\
\text { Lactobacillus, Corynebacterium, } \\
\text { E. coli, Streptococcus and } \\
\text { Pseudomonas }\end{array}$ \\
\hline 1 & $\begin{array}{l}\text { Bacillus, Micrococcus, } \\
\text { Lactobacillus, Pseudomonas, } \\
\text { Corynebacterium and E. coli }\end{array}$ & $\begin{array}{l}\text { Bacillus, Micrococcus, } \\
\text { Lactobacillus, Corynebacterium } \\
\text { and E. coli }\end{array}$ & $\begin{array}{l}\text { Bacillus, Micrococcus, } \\
\text { Corynebacterium and } \\
\text { Pseudomonas }\end{array}$ \\
\hline 8 & $\begin{array}{l}\text { Bacillus, Micrococcus, } \\
\text { Lactobacillus E. coli and } \\
\text { Corynebacterium }\end{array}$ & $\begin{array}{l}\text { Bacillus, Micrococcus, } \\
\text { Lactobacillus and } \\
\text { Corynebacterium }\end{array}$ & $\begin{array}{l}\text { Bacillus, Micrococcus, } \\
\text { Lactobacillus, Pseudomonas } \\
\text { and Corynebacterium }\end{array}$ \\
\hline 15 & $\begin{array}{l}\text { Bacillus, Micrococcus, } \\
\text { Pseudomonas, Lactobacillus } \\
\text { and Corynebacterium }\end{array}$ & $\begin{array}{l}\text { Bacillus, Micrococcus, } \\
\text { Lactobacillus, Pseudomonas and } \\
\text { Corynebacterium }\end{array}$ & $\begin{array}{l}\text { Bacillus, Micrococcus, } \\
\text { Lactobacillus and Pseudomonas }\end{array}$ \\
\hline 22 & $\begin{array}{l}\text { Bacillus, Micrococcus, } \\
\text { Lactobacillus and } \\
\text { Corynebacterium }\end{array}$ & $\begin{array}{l}\text { Bacillus, Micrococcus, } \\
\text { Lactobacillus and } \\
\text { Corynebacterium }\end{array}$ & $\begin{array}{l}\text { Bacillus, Micrococcus and } \\
\text { Lactobacillus }\end{array}$ \\
\hline 30 & $\begin{array}{l}\text { Bacillus, Micrococcus, } \\
\text { Lactobacillus and } \\
\text { Corynebacterium }\end{array}$ & $\begin{array}{l}\text { Bacillus, Micrococcus and } \\
\text { Lactobacillus }\end{array}$ & $\begin{array}{l}\text { Bacillus, Lactobacillus and } \\
\text { Micrococcus }\end{array}$ \\
\hline 45 & $\begin{array}{l}\text { Bacillus, Micrococcus and } \\
\text { Lactobacillus }\end{array}$ & $\begin{array}{l}\text { Bacillus, Micrococcus and } \\
\text { Lactobacillus }\end{array}$ & $\begin{array}{l}\text { Bacillus, Micrococcus and } \\
\text { Lactobacillus }\end{array}$ \\
\hline 60 & $\begin{array}{l}\text { Bacillus, Micrococcus, } \\
\text { Lactobacillus and } \\
\text { Pseudomonas }\end{array}$ & $\begin{array}{l}\text { Bacillus, Micrococcus, } \\
\text { Lactobacillus and Pseudomonas }\end{array}$ & $\begin{array}{l}\text { Bacillus, Micrococcus and } \\
\text { Lactobacillus }\end{array}$ \\
\hline 75 & $\begin{array}{l}\text { Bacillus, Micrococcus, and } \\
\text { Pseudomonas }\end{array}$ & $\begin{array}{l}\text { Bacillus, Micrococcus, and } \\
\text { Pseudomonas }\end{array}$ & $\begin{array}{l}\text { Bacillus, Micrococcus and } \\
\text { Lactobacillus }\end{array}$ \\
\hline 90 & $\begin{array}{l}\text { Bacillus, Micrococcus and } \\
\text { Lactobacillus }\end{array}$ & $\begin{array}{l}\text { Bacillus, Micrococcus and } \\
\text { Lactobacillus }\end{array}$ & $\begin{array}{l}\text { Bacillus, Micrococcus and } \\
\text { Lactobacillus }\end{array}$ \\
\hline 105 & $\begin{array}{l}\text { Bacillus, Micrococcus, } \\
\text { Pseudomonas and } \\
\text { Lactobacillus }\end{array}$ & $\begin{array}{l}\text { Bacillus, Micrococcus and } \\
\text { Lactobacillus }\end{array}$ & $\begin{array}{l}\text { Bacillus, Micrococcus and } \\
\text { Lactobacillus }\end{array}$ \\
\hline
\end{tabular}


Table 1. Generic Distribution of Bacteria in 3 (three) treatments during fermentation (Contd.)

\begin{tabular}{|c|c|c|c|}
\hline 120 & $\begin{array}{l}\text { Bacillus, Micrococcus, } \\
\text { Pseudomonas and } \\
\text { Lactobacillus }\end{array}$ & $\begin{array}{l}\text { Bacillus, Micrococcus, } \\
\text { Pseudomonas and Lactobacillus }\end{array}$ & $\begin{array}{l}\text { Bacillus, Micrococcus and } \\
\text { Lactobacillus }\end{array}$ \\
\hline 135 & $\begin{array}{l}\text { Bacillus, Micrococcus and } \\
\text { Lactobacillus }\end{array}$ & $\begin{array}{l}\text { Bacillus, Micrococcus and } \\
\text { Lactobacillus }\end{array}$ & $\begin{array}{l}\text { Bacillus, Micrococcus and } \\
\text { Lactobacillus }\end{array}$ \\
\hline 150 & $\begin{array}{l}\text { Bacillus, Micrococcus, } \\
\text { Lactobacillus and } \\
\text { Pseudomonas }\end{array}$ & $\begin{array}{l}\text { Bacillus, Micrococcus, } \\
\text { Lactobacillus and Pseudomonas }\end{array}$ & $\begin{array}{l}\text { Bacillus, Micrococcus, } \\
\text { Lactobacillus and Pseudomonas }\end{array}$ \\
\hline 165 & $\begin{array}{l}\text { Bacillus, Lactobacillus, and } \\
\text { Pseudomonas }\end{array}$ & $\begin{array}{l}\text { Bacillus, Micrococcus, } \\
\text { Streptococcus and } \\
\text { Pseudomonas }\end{array}$ & $\begin{array}{l}\text { Bacillus, Micrococcus, } \\
\text { Streptococcus and } \\
\text { Pseudomonas }\end{array}$ \\
\hline 180 & $\begin{array}{l}\text { Bacillus, Micrococcus, } \\
\text { Lactobacillus and } \\
\text { Pseudomonas }\end{array}$ & $\begin{array}{l}\text { Bacillus, Micrococcus, } \\
\text { Lactobacillus and Pseudomonas }\end{array}$ & $\begin{array}{l}\text { Bacillus, Micrococcus, } \\
\text { Lactobacillus and Pseudomonas }\end{array}$ \\
\hline 195 & $\begin{array}{l}\text { Bacillus, Micrococcus, } \\
\text { Lactobacillus and } \\
\text { Pseudomonas }\end{array}$ & $\begin{array}{l}\text { Bacillus, Micrococcus, } \\
\text { Lactobacillus and Pseudomonas }\end{array}$ & $\begin{array}{l}\text { Bacillus, Micrococcus, } \\
\text { Lactobacillus and Pseudomonas }\end{array}$ \\
\hline 210 & $\begin{array}{l}\text { Bacillus, Micrococcus, } \\
\text { Lactobacillus and } \\
\text { Pseudomonas }\end{array}$ & $\begin{array}{l}\text { Bacillus, Micrococcus, } \\
\text { Lactobacillus and Pseudomonas }\end{array}$ & $\begin{array}{l}\text { Bacillus, Micrococcus, } \\
\text { Lactobacillus and Pseudomonas }\end{array}$ \\
\hline 240 & $\begin{array}{l}\text { Bacillus, Micrococcus, } \\
\text { Lactobacillus and } \\
\text { Pseudomonas }\end{array}$ & $\begin{array}{l}\text { Bacillus, Micrococcus, } \\
\text { Lactobacillus and Pseudomonas }\end{array}$ & $\begin{array}{l}\text { Bacillus, Micrococcus, } \\
\text { Lactobacillus and Pseudomonas }\end{array}$ \\
\hline 270 & $\begin{array}{l}\text { Bacillus, Micrococcus, } \\
\text { Lactobacillus and } \\
\text { Pseudomonas }\end{array}$ & $\begin{array}{l}\text { Bacillus, Micrococcus, } \\
\text { Lactobacillus and Pseudomonas }\end{array}$ & $\begin{array}{l}\text { Bacillus, Micrococcus, } \\
\text { Lactobacillus and Pseudomonas }\end{array}$ \\
\hline $\begin{array}{l}\text { Final Fish } \\
\text { Sauce (After } \\
15 \text { days of } \\
\text { aging and } \\
\text { filtration) }\end{array}$ & $\begin{array}{l}\text { Bacillus, Micrococcus, } \\
\text { Lactobacillus and } \\
\text { Pseudomonas }\end{array}$ & $\begin{array}{l}\text { Bacillus, Micrococcus, } \\
\text { Lactobacillus and Pseudomonas }\end{array}$ & $\begin{array}{l}\text { Bacillus, Micrococcus, } \\
\text { Lactobacillus and Pseudomonas }\end{array}$ \\
\hline
\end{tabular}

*Treatment-I- 25\% salted fish, Treatment-II- 30\% salted fish, Treatment-III- 35\% salted fish

\section{Panel test of fish sauce}

After aging and filtration the physical quality of final fish sauce in 3 (three) different treatments was judged through panel test and the result has been represented in Table 3. Colour of the fish sauce in treatment-I was reddish brown, whereas treatment-II and treatment-III had deep red and blackish red colours respectively. The aroma of treatment-I was light fishy sweet flavor, but the rest two treatments had strong fishy flavor. Fish sauce of treatment-I was more acceptable than other 2 (two) treatments due to its less saltiness and also the overall appearance was perfectly matched with the imported fish sauce available in the market. Taniguchi and Takano (2007) stated that high-quality salmon sauce was developed with mature chum salmon (Oncorhynchus keta) samples, were eviscerated, deboned, washed, cut in small pieces, added $25 \%$ salt and internal organs. This result matched with the present study. 
Table 2. Physical changes in 3 (Three) Treatments during fermentation

\begin{tabular}{|c|c|c|c|c|c|c|c|c|c|}
\hline \multirow{2}{*}{ Days } & \multicolumn{3}{|c|}{ Outer Appearance } & \multicolumn{3}{|c|}{ Texture of fish body } & \multicolumn{3}{|c|}{ Colour of Exudates } \\
\hline & Treatment-I* & Treatment-II* & Treatment-III* & Treatment-I* & Treatment-II* & Treatment-III* & Treatment-I* & Treatment-II* & Treatment-III* \\
\hline 0 & Fresh fish & Fresh fish & Fresh fish & Hard & Hard & Hard & No Exudates & No Exudates & No Exudates \\
\hline 1 & $\begin{array}{l}\text { Fresh like } \\
\text { appearance }\end{array}$ & $\begin{array}{l}\text { Fresh like } \\
\text { appearance }\end{array}$ & $\begin{array}{l}\text { Fresh like } \\
\text { appearance }\end{array}$ & Hard & Hard & Hard & Light yellowish & Light yellowish & Light yellowish \\
\hline 15 & Less shiny & Less shiny & Less shiny & Hard & Hard & Hard & Yellowish & Yellowish & Yellowish \\
\hline 30 & Loss of shine & Loss of shine & Loss of shine & Slight soft & Slight soft & Slight soft & Light reddish & Light reddish & Light reddish \\
\hline 45 & $\begin{array}{l}\text { Complete loss } \\
\text { of shine }\end{array}$ & $\begin{array}{l}\text { Complete loss of } \\
\text { shine }\end{array}$ & $\begin{array}{l}\text { Complete loss } \\
\text { of shine }\end{array}$ & Slight soft & Slight soft & Slight soft & Reddish & Reddish & Reddish \\
\hline 60 & $\begin{array}{l}\text { Complete loss } \\
\text { of shine }\end{array}$ & $\begin{array}{l}\text { Complete loss of } \\
\text { shine }\end{array}$ & $\begin{array}{l}\text { Complete loss } \\
\text { of shine }\end{array}$ & $\begin{array}{l}\text { Belly portion more } \\
\text { soft }\end{array}$ & $\begin{array}{l}\text { Belly portion more } \\
\text { soft }\end{array}$ & $\begin{array}{l}\text { Belly portion more } \\
\text { soft }\end{array}$ & Dark red & Dark red & Dark red \\
\hline 75 & $\begin{array}{l}\text { Few scale } \\
\text { start to loss }\end{array}$ & Scale intact & Scale intact & $\begin{array}{l}\text { Belly portion more } \\
\text { soft }\end{array}$ & $\begin{array}{l}\text { Belly portion more } \\
\text { soft }\end{array}$ & $\begin{array}{l}\text { Belly portion more } \\
\text { soft }\end{array}$ & $\begin{array}{l}\text { Red and slight } \\
\text { turbid }\end{array}$ & $\begin{array}{l}\text { Red and slight } \\
\text { turbid }\end{array}$ & Dark red \\
\hline 90 & $\begin{array}{l}\text { More scale } \\
\text { loss }\end{array}$ & $\begin{array}{l}\text { Few scale start } \\
\text { to loss }\end{array}$ & $\begin{array}{l}\text { Few scale } \\
\text { start to loss }\end{array}$ & $\begin{array}{l}\text { Belly flaps start to } \\
\text { digest }\end{array}$ & $\begin{array}{l}\text { Belly flaps start to } \\
\text { digest }\end{array}$ & $\begin{array}{l}\text { Belly portion more } \\
\text { soft }\end{array}$ & Turbid red & Turbid red & $\begin{array}{l}\text { Red and slight } \\
\text { turbid }\end{array}$ \\
\hline 105 & $\begin{array}{l}30 \% \text { scale } \\
\text { loss }\end{array}$ & $15 \%$ scale loss & $\begin{array}{l}10 \% \text { scale } \\
\text { loss }\end{array}$ & Stomach digested & Stomach digested & $\begin{array}{l}\text { Belly flaps start to } \\
\text { digest }\end{array}$ & $\begin{array}{l}\text { Turbid and deep } \\
\text { red }\end{array}$ & $\begin{array}{l}\text { Turbid and deep } \\
\text { red }\end{array}$ & Turbid red \\
\hline 120 & $\begin{array}{l}45 \% \text { scale } \\
\text { loss }\end{array}$ & $30 \%$ scale loss & $\begin{array}{l}25 \% \text { scale } \\
\text { loss }\end{array}$ & Stomach digested & Stomach digested & $\begin{array}{l}\text { Belly flaps start to } \\
\text { digest }\end{array}$ & $\begin{array}{l}\text { Turbid and deep } \\
\text { red }\end{array}$ & $\begin{array}{l}\text { Turbid and deep } \\
\text { red }\end{array}$ & Turbid red \\
\hline 135 & $\begin{array}{l}55 \% \text { scale } \\
\text { loss }\end{array}$ & $42 \%$ scale loss & $\begin{array}{l}35 \% \text { scale } \\
\text { loss }\end{array}$ & $\begin{array}{l}\text { Belly flaps } \\
\text { digested }\end{array}$ & $\begin{array}{l}\text { Belly flaps } \\
\text { digested }\end{array}$ & Stomach digested & Deep turbid red & Deep turbid red & Deep turbid red \\
\hline 150 & $\begin{array}{l}72 \% \text { scale } \\
\text { loss }\end{array}$ & $65 \%$ scale loss & $\begin{array}{l}50 \% \text { scale } \\
\text { loss }\end{array}$ & $\begin{array}{l}\text { Belly flaps } \\
\text { digested }\end{array}$ & $\begin{array}{l}\text { Belly flaps } \\
\text { digested }\end{array}$ & Stomach digested & Deep turbid red & Deep turbid red & Deep turbid red \\
\hline 165 & $\begin{array}{l}85 \% \text { scale } \\
\text { loss }\end{array}$ & $75 \%$ scale loss & $\begin{array}{l}60 \% \text { scale } \\
\text { loss }\end{array}$ & $\begin{array}{l}\text { Muscle near the } \\
\text { belly become } \\
\text { more soft }\end{array}$ & $\begin{array}{l}\text { Muscle near the } \\
\text { belly become } \\
\text { more soft }\end{array}$ & $\begin{array}{l}\text { Belly flaps } \\
\text { digested }\end{array}$ & Deep turbid red & Deep turbid red & Deep turbid red \\
\hline 180 & $\begin{array}{l}\text { Scale } \\
\text { completely } \\
\text { loss }\end{array}$ & $90 \%$ scale loss & $\begin{array}{l}80 \% \text { scale } \\
\text { loss }\end{array}$ & $\begin{array}{l}\text { Complete body } \\
\text { become more soft }\end{array}$ & $\begin{array}{l}\text { Complete body } \\
\text { become more soft }\end{array}$ & $\begin{array}{l}\text { Muscle near the } \\
\text { belly digested }\end{array}$ & Deep turbid red & Deep turbid red & Deep turbid red \\
\hline
\end{tabular}


Table 2. Physical changes in 3 (Three) Treatments during fermentation (Contd.)

\begin{tabular}{|c|c|c|c|c|c|c|c|c|c|}
\hline 195 & $\begin{array}{l}\text { Skin start to } \\
\text { loss }\end{array}$ & $\begin{array}{l}\text { Scale } \\
\text { completely loss }\end{array}$ & $\begin{array}{l}95 \% \text { scale } \\
\text { loss }\end{array}$ & $\begin{array}{l}\text { Muscle of other } \\
\text { portion start to } \\
\text { digest }\end{array}$ & $\begin{array}{l}\text { Muscle of other } \\
\text { portion start to } \\
\text { digest }\end{array}$ & $\begin{array}{l}\text { Complete body } \\
\text { become more soft }\end{array}$ & Highly turbid red & Highly turbid red & Highly turbid red \\
\hline 210 & $\begin{array}{l}\text { Most of skin } \\
\text { part loss }\end{array}$ & Skin start to loss & $\begin{array}{l}\text { Scale } \\
\text { completely } \\
\text { loss }\end{array}$ & $\begin{array}{l}\text { About } 30 \% \text { muscle } \\
\text { digested }\end{array}$ & $\begin{array}{l}\text { About } 20 \% \text { muscle } \\
\text { digested }\end{array}$ & $\begin{array}{l}\text { Muscle of other } \\
\text { portion start to } \\
\text { digest }\end{array}$ & Highly turbid red & Highly turbid red & $\begin{array}{l}\text { Highly turbid } \\
\text { red }\end{array}$ \\
\hline 225 & Flesh exposed & $\begin{array}{l}\text { Most of skin part } \\
\text { loss }\end{array}$ & $\begin{array}{l}\text { Skin start to } \\
\text { loss }\end{array}$ & $\begin{array}{l}\text { About } 65 \% \text { muscle } \\
\text { digested }\end{array}$ & $\begin{array}{l}\text { About } 55 \% \text { muscle } \\
\text { digested }\end{array}$ & $\begin{array}{l}\text { About 35\% muscle } \\
\text { digested }\end{array}$ & $\begin{array}{l}\text { Highly turbid red } \\
\text { with flesh parts }\end{array}$ & $\begin{array}{l}\text { Highly turbid red } \\
\text { with flesh parts }\end{array}$ & $\begin{array}{l}\text { Highly turbid red } \\
\text { with flesh parts }\end{array}$ \\
\hline 240 & $\begin{array}{l}\text { Bones } \\
\text { exposed }\end{array}$ & Bones exposed & $\begin{array}{l}\text { Bones } \\
\text { exposed }\end{array}$ & $\begin{array}{l}\text { About } 90 \% \text { muscle } \\
\text { digested }\end{array}$ & $\begin{array}{l}\text { About } 82 \% \text { muscle } \\
\text { digested }\end{array}$ & $\begin{array}{l}\text { About } 63 \% \text { muscle } \\
\text { digested }\end{array}$ & $\begin{array}{l}\text { Highly turbid } \\
\text { with flesh parts }\end{array}$ & $\begin{array}{l}\text { Highly turbid red } \\
\text { with flesh parts }\end{array}$ & $\begin{array}{l}\text { Highly turbid red } \\
\text { with flesh parts }\end{array}$ \\
\hline 255 & $\begin{array}{l}\text { Bones fully } \\
\text { exposed }\end{array}$ & Bones exposed & $\begin{array}{l}\text { Bones } \\
\text { exposed }\end{array}$ & $\begin{array}{l}\text { Muscle fully } \\
\text { digested }\end{array}$ & $\begin{array}{l}\text { About 95\% muscle } \\
\text { digested }\end{array}$ & $\begin{array}{l}\text { About } 85 \% \text { muscle } \\
\text { digested }\end{array}$ & $\begin{array}{l}\text { Highly turbid } \\
\text { with dissolved } \\
\text { flesh }\end{array}$ & $\begin{array}{l}\text { Highly turbid red } \\
\text { with dissolved } \\
\text { flesh }\end{array}$ & $\begin{array}{l}\text { Highly turbid } \\
\text { dark red with } \\
\text { flesh parts }\end{array}$ \\
\hline 270 & $\begin{array}{l}\text { Bones fully } \\
\text { exposed }\end{array}$ & $\begin{array}{l}\text { Bones fully } \\
\text { exposed }\end{array}$ & $\begin{array}{l}\text { Bones fully } \\
\text { exposed }\end{array}$ & $\begin{array}{l}\text { Muscle fully } \\
\text { digested }\end{array}$ & $\begin{array}{l}\text { Muscle fully } \\
\text { digested }\end{array}$ & $\begin{array}{l}\text { About } 98 \% \text { muscle } \\
\text { digested }\end{array}$ & $\begin{array}{l}\text { Highly turbid red } \\
\text { with dissolved } \\
\text { flesh }\end{array}$ & $\begin{array}{l}\text { Highly turbid } \\
\text { dark red with } \\
\text { dissolved flesh }\end{array}$ & $\begin{array}{l}\text { Highly turbid } \\
\text { dark red with } \\
\text { dissolved flesh }\end{array}$ \\
\hline
\end{tabular}

${ }^{\star}$ Treatment-I- 25\% salted fish, Treatment-II- 30\% salted fish, Treatment-III- 35\% salted fish 
Table 3. Panel test report of fish sauce

\begin{tabular}{llll}
\hline Parameters & Treatment-I* $^{*}$ & Treatment-II* $^{*}$ & Treatment-III* $^{*}$ \\
\hline Colour & Reddish brown & Deep Red & Blackish red colour \\
Flavour & Light fishy sweet flavour & Strong fishy flavour & Strong fishy flavor \\
Test & Salty & More salty & Highly salty \\
Clearness & Clear & Slight cloudy & More cloudy \\
Comparison with & More similar & Less similar & Less similar \\
imported fish sauce & & & \\
available in the market & & & \\
Acceptability & Highly acceptable & Acceptable & Less acceptable \\
\hline
\end{tabular}

*Treatment-I- 25\% salted fish, Treatment-II- 30\% salted fish, Treatment-III- 35\% salted fish

\section{CONCLUSION}

Based on above discussion it can be concluded that fish sauce was prepared due to combined action of enzymes present in fish body and microorganisms, where salt worked as a preservative. Moreover only the salt tolerant bacteria can survive in this long period of fermentation. Considering the result of physical parameters of fish sauce it can be declared that fish sauce prepared with $25 \%$ salt (Treatment-I) was found to be the best quality.

\section{REFERENCES}

1. Barrow GI and RKA Feltham, 1993. Cowan and Steel's Manual for the Identification of Medical Bacteria. $3^{\text {rd }}$ Ed. Cambridge University Press. pp. 1- 331.

2. Beddows CG, 1985. Microbiology of Fermented Foods. Vol. II. Edited by B.J.B. Wood. Elsevier Applied Science Publishers, London.

3. Beddows CG, AG Ardeshir and WJ Daud, 1979. Biochemical changes occurring during the manufacture of Budu. Journal of Science Food and Agriculture, 30: 1097-1103.

4. Crisan EV and A Sands, 1975. Microflora of four fermented fish sauces. Journal of Applied Microbialogy, 29: 106-108.

5. FAO 1979. Manuals of food quality control, 4-Rev.1- Microbiological Analysis, Food and Agriculture Organization of the United Nations, Rome. pp. 14.

6. Lopetcharat $\mathrm{K}$ and JW Park, 2002. Characteristics of fish sauce made from Pacific whiting and surimi by-products during fermentation stage. Journal of Food Science, 67: 511-516.

7. Mclver RC, RI Brooks and GA Reineccius, 1982. Flavor of fermented fish sauce. Journal of Agricultural Food chemistry, 30: 1017-1020.

8. Orejana FM, 1978. Proteolysis and Control mechanism in fish saucefermentation. PhD. Thesis. University of Washington, Seattle, Washington. pp. 230.

9. Saisithi $\mathrm{P}$, 1967. Studies on the origins and development of typical flavor and aroma of thai fish sauce. Ph.D. Thesis. University of Washington, Seattle, Washington. pp. 173.

10. Saisithi P, B Kasemsarn, J Liston and AM Dollar, 1966. Microbiology and Chemistry of fermented fish. Journal of Food Science, 31: 105-10.

11. Sanceda N, E Suzuki and T Kurata, 2003. Quality and sensory acceptance of fish sauce partially substituting sodium chloride or natural salt with potassium chloride during the fermentation process. International Journal of Food Science, 38: 435-443,

12. Sanchez PC, 2001. Microorganisms in traditional fermented foods: resources for biotechnological development. Microbial Diversity in Asia. pp. 143-157. 
13. Taniguchi AY and K Takano, 2007. Effects of protease and collagenase in the production of fish sauce using mature chum salmon (Oncorhynchus keta). Journal of World Aquaculture, 38: 19-21.

14. Thongthai $C$ and $M$ Siriwongpairat, 1990. The sequential quantization of microorganisms in traditionally fermented fish sauce (Nampla). In "Post-Harvest Technology, Preservation and Quality of Fish in South East Asia. Ed. by PJA Reilly, RWH Parry and LE Barile. 51-9. International Foundation of Science, Stockholm. pp. 126-132.

15. Visessanguan W, S Benjakul, S Riebroy and P Thepksikul, 2004. Changes in composition and functional properties of proteins and their contributions to Nham characteristics. Journal of Food Chemistry, 66: 579-588.

16. Zaman MZ, AS Abdulamir, AF Bakar, J Selamat and J Bakar, 2009. Microbiological, physicochemical and health impact of high level of biogenic amines in fish sauce. Journal of Applied Science, 6: 11991211. 